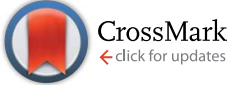

Cite this: Chem. Sci., 2015, 6, 6097

\title{
Coupling of chromophores with exactly opposite luminescence behaviours in mesostructured organosilicas for high-efficiency multicolour emission $\dagger$
}

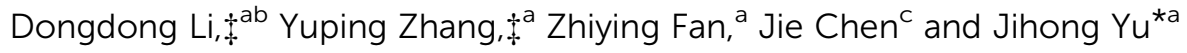

Aggregation-induced emission (AIE) and aggregation-caused quenching (ACQ) materials are important for various fluorescence-based applications but cannot easily collaborate because of their opposite luminescence behaviours. Here, we demonstrate a strategy to integrate AIE and ACQ chromophores in periodic mesoporous organosilicas (PMOs) for high-efficiency multicolour emission. Tetraphenylethene (TPE)-bridged AIE-PMOs are prepared as hosts to encapsulate ACQ dyes (e.g. RhB), which enables finetuning of ACQ@AIE-PMO emissions over the entire visible spectrum in the solid and film states. Significantly, high-quality white light is achieved with CIE coordinates of $(0.32,0.33)$ and a quantum yield of up to $49.6 \%$. Because of their high stability and solution processability, the ACQ@AIE-PMOs can be applied in solid-state lighting and bioimaging. This design concept opens up new perspectives for developing high-performance luminescent materials by the combination of a wide variety of AIE and $A C Q$ chromophores.
\end{abstract}

Received 8th June 2015

Accepted 20th July 2015

DOI: $10.1039 /$ c5sc02044a

www.rsc.org/chemicalscience

intramolecular rotations (RIR). ${ }^{4}$ Because of their exactly opposite luminescent behaviours, AIE and ACQ materials are not easily coupled to construct new efficient luminescent materials. Some attempts were made to combine AIE and ACQ molecules by covalent bonding, ${ }^{5}$ but they failed in realising the advantages of combining both classes of dye. The development of a feasible approach for achieving the effective collaboration of AIE and ACQ dyes and constructing highly efficient and tuneable multicolour-emission materials for practical applications remains an important challenge.

Solid-state light-emitting materials, particularly whitelighting materials, have aroused considerable interest in recent years. ${ }^{6}$ Luminescent periodic mesoporous organosilicas (PMOs) are an interesting class of inorganic-organic hybrid materials with excellent thermal, mechanical and photochemical stabilities, in which the chromophores can be densely and covalently embedded within the framework forming the pore walls. ${ }^{7}$ So far, the reported fluorescent PMO materials are mainly based on ACQ bridging molecules. ${ }^{8} \mathrm{~A}$ high chromophore concentration in the pore walls results in relatively low fluorescence quantum yields compared to those of their precursors because they tend ${ }^{a}$ State Key Laboratory of Inorganic Synthesis and Preparative Chemistry, College of Chemistry, Jilin University, Changchun, 130012, P. R. China. E-mail: jihong@jlu. edu.cn

${ }^{b}$ Department of Materials Science, Jilin University, Changchun, 130012, P. R. China ${ }^{c}$ Key Laboratory of Polymer Ecomaterials, Changchun Institute of Applied Chemistry, Chinese Academy of Sciences, Changchun, 130022, China

$\dagger$ Electronic supplementary information (ESI) available: Experimental details for the synthesis, TEM, SEM, XRD patterns, FTIR spectra, absorption and PL spectra. See DOI: 10.1039/c5sc02044a

\$ These authors contributed equally to this work. materials with highly efficient emission suitable for solid-state lighting applications is required. Recently, we developed AIEluminogen-functionalised mesoporous materials using postgrafting methods; ${ }^{\mathbf{1 0}}$ their emission intensities were enhanced with increased loading amounts of the AIE-active unit. It is believed that the introduction of AIE chromophores instead of 
conventional dyes as the bridging groups is an efficient approach to construct high-performance luminescent PMOs. Significantly, the ordered channels of AIE-unit-embedded PMOs can also host a variety of chromophores and stabilize their optical properties, which is advantageous for the fabrication of new donor-acceptor systems that allow the coupling of AIE and ACQ dyes on the basis of efficient fluorescence resonance energy transfer (FRET). In addition, the pore size of PMOs located in the working distances of photoinduced electron transfer and/or energy transfer can dramatically enhance the electron transfer efficiency. ${ }^{\mathbf{1 1}}$ We expect that such unique features of mesostructured organosilicas will not only afford great advantages for enhancing the luminescence of the AIE chromophores in the mesoporous framework but also lead to multicolour emission, including the emission of pure white light, upon encapsulation of ACQ dyes in the mesochannels.

Here, we introduce an efficient strategy to achieve the coupling of AIE and ACQ chromophores in PMOs via FRET for the construction of high-efficiency and tuneable multicolouremission materials (Fig. 1). Blue-emitting TPE-bridged organosilane is used as a precursor for preparing high-performance luminescent PMOs, which avoid the typical fluorescence quenching suffered by ACQ dyes; these AIE-PMOs are employed as energy donors. Conventional ACQ dyes, such as rhodamine B (RhB) and rhodamine 6G (R6G), are encapsulated in the pores of the AIE-PMOs and used as energy acceptors. The resulting ACQ@AIE-PMO composites show multicolour emission over a wide range of the visible spectrum with high quantum yields. Significantly, highly pure solid-state white-light emission can be obtained with a quantum yield as high as $49.6 \%$, which is superior to the level of some reported inorganic-organic hybrid

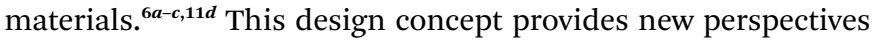
for developing high-performance luminescent materials by the combination of a wide variety of AIE and ACQ chromophores for

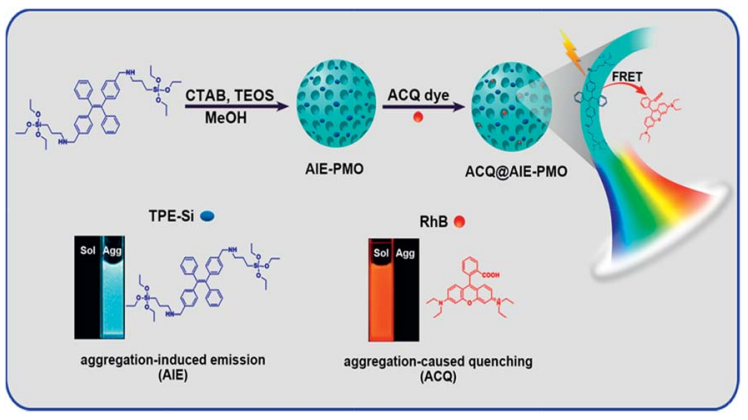

Fig. 1 Schematic illustration of the construction of ACQ@AIE-PMO with tuneable multicolour emission. AIE-PMO nanospheres are prepared by using AIE-active TPE-Si as a precursor, where TPE units are covalently embedded within the framework forming the pore walls; $A C Q$ molecules (such as $\mathrm{RhB}$ ) are then encapsulated in the mesoporous channels of the AIE-PMO; tuneable multicolour emission of PMO is achieved on the basis of FRET from the AIE-PMO donor to the ACQ acceptor. Note that the TPE-Si precursor is nonemissive in solution but luminesces intensively upon molecular aggregation, while $\mathrm{RhB}$ dye is emissive in solution but suffers from aggregation-caused fluorescence quenching. fluorescence-based applications in solid-state lighting, biomedicine and other areas.

\section{Results and discussion}

\section{Synthesis and characterisation of TPE-bridged PMOs}

The AIE-luminogen-containing organosilica (TPE-Si) precursor was prepared by reacting 3-aminopropyltriethoxysilane (APTS) with 1,2-bis[4-(bromomethyl)phenyl]-1,2-diphenylethene (BTPE) without further purification. The presence of the TPE-Si precursor was proved by mass spectroscopic (MS) data. It emits strong blue luminescence in a $\mathrm{THF} / \mathrm{H}_{2} \mathrm{O}$ mixture with $90 \%$ water under UV light illumination (365 nm), showing a typical AIE character (Fig. S1†). AIE-PMOs with different amounts of organosilica were then synthesised by basic hydrolysis and polycondensation of TPE-Si with TEOS in the presence of cetyltrimethylammonium bromide (CTAB) surfactant. The concentrations of the TPE unit introduced into the materials were approximately $0.12,0.17$ and $0.21 \mathrm{mmol} \mathrm{g}^{-1}$, as determined by elemental analysis (Table S1 $\dagger$ ); the resulting materials are denoted as AIE-PMO1, AIE-PMO2 and AIE-PMO3, respectively. The ordered 2D hexagonal mesostructures of the AIE-PMO nanoparticles were confirmed by small-angle powder X-ray diffraction (XRD) (Fig. 2a), scanning electron microscopy (SEM) (Fig. 2b and S2 $\dagger$ ), nitrogen adsorption-desorption isotherms (Fig. 2c) and transmission electron microscopy (TEM) (Fig. 2d and $\mathrm{S} 3 \dagger$ ). When the amount of TPE-Si unit embedded in the PMOs was increased, the $d$ values of the AIE-PMOs slightly increased, perhaps because the introduction of more TPE molecules partially reduced the pore size, as shown in Fig. 2a and c. On the other hand, as TPE-Si is a hydrophobic molecule, the CTAB surfactant micelles will be changed with the introduction of more TPE-Si, which may further affect their morphology even to the point of forming a spherical morphology. ${ }^{12}$ The bands at $1400-1600 \mathrm{~cm}^{-1}$ in the Fourier transform-infrared (FT-IR) spectra attributed to the $\mathrm{C}=\mathrm{C}$ stretching vibration of aromatic rings confirm that the AIE-active TPE molecules were covalently embedded within the framework forming the pore walls (Fig. S4†)..$^{13}$

All of the AIE-PMO materials exhibited strong fluorescence centred at approximately $480 \mathrm{~nm}$ (Fig. 3a). The emission behaviour is attributed primarily to the ability of the rigid framework of the mesostructured materials to greatly restrict the intramolecular rotations of the embedded TPE molecules by covalent bonding, thus blocking the nonradiative relaxation channel, and to populate radiative decay to the ground state, making the materials highly emissive. ${ }^{4 b}$ Furthermore, the photoluminescence (PL) intensity was noticeably enhanced with increased embedding amount of the TPE chromophores, and their quantum yields are $18.1 \%, 20.0 \%$ and $26.9 \%$ for AIE-PMO1, AIE-PMO2 and AIE-PMO3, respectively.

\section{Tuneable multicolour emission of RhB-doped AIE-PMOs}

A new donor-acceptor system of ACQ@AIE-PMO was constructed by using the blue-emitting AIE-PMOs as energy donors and the encapsulated ACQ dyes as energy acceptors. As a model 

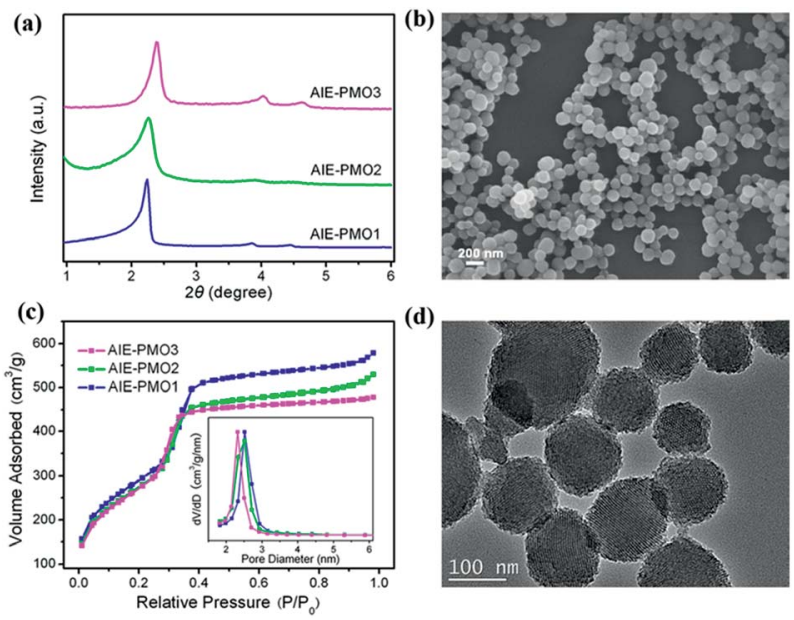

Fig. 2 (a) Powder XRD patterns of mesostructured AIE-PMOs. (b) SEM image of AIE-PMO3. (c) $\mathrm{N}_{2}$ adsorption-desorption isotherms of AIE-PMOs and their corresponding pore size distributions. (d) TEM image of AIE-PMO3.
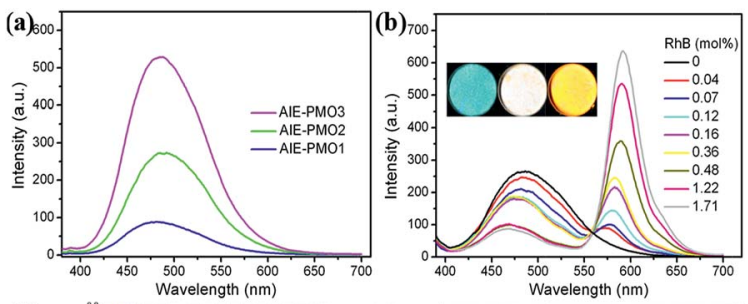

(c)
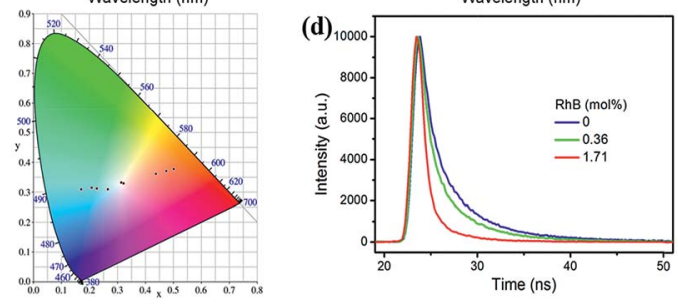

Fig. 3 (a) Fluorescence spectra of AlE-PMOs in the solid state. (b) Fluorescence spectra of RhB@AIE-PMO2 powders with different RhB contents. (c) Corresponding emission colours of RhB@AlE-PMO2 powders (marked by the dots) in the CIE 1931 chromaticity diagram. (d) Fluorescence decay profiles of RhBQAIE-PMO2 with different RhB contents at $\lambda_{\mathrm{ex}}=365 \mathrm{~nm}$ and $\lambda_{\mathrm{em}}=480 \mathrm{~nm}$.

system for demonstrating the utility and validity of this approach, a red-emitting RhB dye was selected because of the sufficient spectral overlap of its absorption band with the emission bands of the AIE-PMOs (Fig. S5 $\dagger$ ). Owing to the similar structure and emission property of the AIE-PMOs, AIE-PMO2 was chosen as a host for the encapsulation of RhB. Powder samples of AIE-PMO2 were first immersed in aqueous solutions with different RhB concentrations. After $24 \mathrm{~h}$, the soaked samples were removed from solution and thoroughly washed with water. As the concentrations of the encapsulated RhB dye increased, the emission of AIE-PMO2 at approximately $480 \mathrm{~nm}$ gradually decreased, and an emission band at approximately $576 \mathrm{~nm}$ attributed to the RhB appeared and increased in intensity under excitation at $365 \mathrm{~nm}$ (Fig. 3b). Meanwhile, the average emission lifetime value of the TPE unit at $480 \mathrm{~nm}$ in AIE-PMO2 was gradually shortened from $4.63 \mathrm{~ns}$ to $3.84 \mathrm{~ns}$ to $1.91 \mathrm{~ns}$, with the increased doping amount of RhB from $0 \mathrm{~mol} \%$ to $0.36 \mathrm{~mol} \%$ to $1.71 \mathrm{~mol} \%$ (with respect to the amount of TPE unit) (Fig. 3d and Table S2 $\dagger$ ). These results suggest an energy transfer from the AIE-PMO framework to the encapsulated RhB dyes. The emission colour of the RhB@AIE-PMO2 powders clearly changed from blue to red via white light under UV irradiation. The Commission International de I'Eclairage (CIE) coordinates were $(0.17,0.28),(0.32,0.33)$ and $(0.50,0.42)$ for an RhB content of $0 \mathrm{~mol} \%, 0.36 \mathrm{~mol} \%$ and $1.71 \mathrm{~mol} \%$, respectively (Fig. 3c). The quantum yield of RhB@AIE-PMO2 under excitation at $365 \mathrm{~nm}$ increased with increasing $\mathrm{RhB}$ content from $20.0 \%$ (blue) to $49.6 \%$ (white) to $80.1 \%$ (red). The enhancement of the quantum yield shows that energy transfer can occur directly from the AIE unit in the framework to the RhB dye in the channels without a radiation-reabsorption process. Significantly, the resulting white light was very close to pure white light with CIE coordinates of $(0.33,0.33)$, and RhB@AIE-PMO2 exhibited a high quantum yield compared with the white-lightemitting inorganic-organic hybrid materials reported to date, such as MOFs, PMOs, nanomaterials and semiconductor bulk materials (Table S3†). ${ }^{\boldsymbol{6 a - c , 1 1 d}}$ Other mesoporous silica, such as SBA-15, can also be used as a host for coupling the AIE and ACQ dyes (Fig. S6†).

The optical stability of RhB@AIE-PMO2 was investigated. After continuous irradiation of the solid sample containing $0.36 \mathrm{~mol} \% \mathrm{RhB}$ with $365 \mathrm{~nm}$ UV light for $45 \mathrm{~min}$, the emission spectral profiles remained approximately identical (Fig. S7†). Meanwhile, the time-dependent emission spectra of RhB@AIE$\mathrm{PMO} 2$ powder dispersed in water also showed no obvious change (Fig. S8 $\dagger$ ). After 24 hours, less than $0.2 \%$ RhB was detected with UV-vis spectroscopic measurement due to the slight desorption from RhB@AIE-PMO2 in aqueous solution (Fig. S9†). These results indicate the excellent stability of RhB@AIE-PMO materials in the solid state and in solution.

The bioimaging abilities of the RhB@AIE-PMO nanoparticles were evaluated with HeLa cells for in vitro cellular imaging. After the cells were incubated with RhB@AIE-PMO2 samples with different $\mathrm{RhB}$ concentrations $(0 \mathrm{~mol} \%$, blue; $0.36 \mathrm{~mol} \%$, white; $1.71 \mathrm{~mol} \%$, red) for $4 \mathrm{~h}$ at $37^{\circ} \mathrm{C}$, strong blue, white and red light emissions, respectively, were observed under the confocal microscope (Fig. 4). The bright-field and fluorescent images of the HeLa cells clearly demonstrate that these luminescent nanoparticles were successfully internalized into the cells, indicating their potential applications in biomedicine.

\section{Tuneable multicolour emission of RhB-doped AIE-PMO films}

We further fabricated PMO composite films with different doping amounts of RhB dye by an acidic sol-gel one-step polycondensation via evaporation-induced self-assembly processes in the presence of P123 surfactant (Fig. S10 $\dagger$ ). The mesoporous structure of the prepared AIE-PMO film was confirmed by XRD (Fig. S11 $\dagger$ ) and TEM analysis (Fig. S12 $\dagger$ ). 


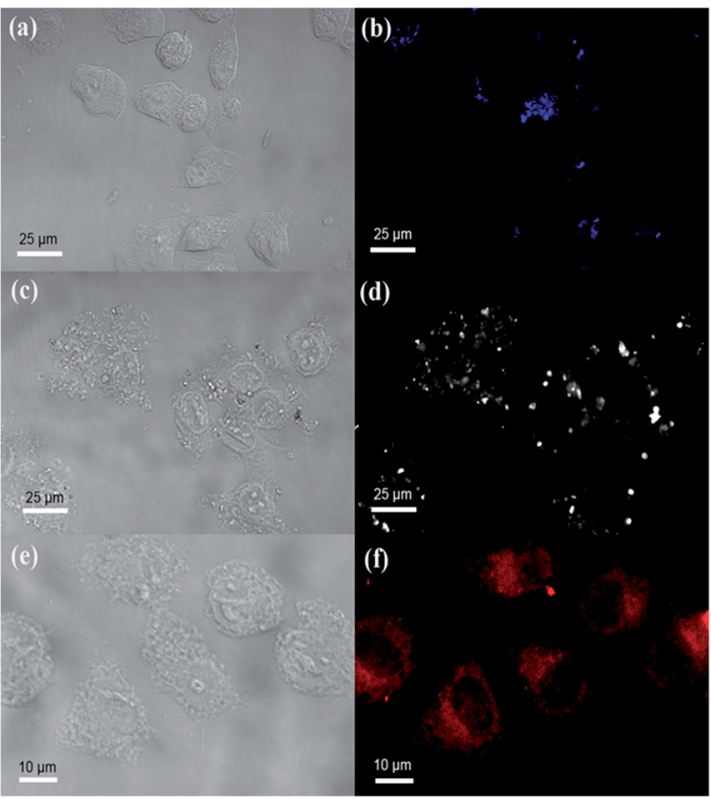

Fig. 4 (Left) Bright-field and (right) fluorescent images of HeLa cells after incubation with RhB aAlE-PMO2 with different RhB concentrations: ( $a$ and b) 0 mol\%, (c and d) $0.36 \mathrm{~mol} \%$, and (e and f) $1.71 \mathrm{~mol} \%$.

Doping of RhB dye into the mesostructured AIE-PMOs enabled flexible tuning of the fluorescence emission colour of the films within a wide range of the visible spectrum. As shown in Fig. 5a, the emission at approximately $574 \mathrm{~nm}$, which was attributed to the doped RhB dye, appeared and increased in intensity under excitation at $365 \mathrm{~nm}$. In addition, the emission intensity of TPE at approximately $474 \mathrm{~nm}$ decreased when the RhB content was increased from 0 to $5.0 \mathrm{~mol} \%$, indicating efficient energy transfer from the AIE-PMO framework to the guest RhB dye. The emission colour of the PMO composite films changed from blue, with CIE coordinates of $(0.16,0.24)$, to yellow-red (0.42, 0.41) (Fig. 5b). More importantly, the RhB@AIE-PMO film containing $3.2 \mathrm{~mol} \% \mathrm{RhB}$ exhibited a high-quality white-light emission with CIE coordinates of $(0.31,0.33)$. Remarkably, the RhB@AIE-PMO films were highly transparent, which is crucial for avoiding the loss of incident light via scattering. Moreover, the machinability of the sol solution for the preparation of PMOs further facilitated drop-casting onto various substrates, as typically used for various assemblies. Fig. 5c shows a blueemitting transparent thin film of AIE-PMO fabricated on a largearea glass substrate, and a white-light emitting film of RhB@AIE-PMO deposited directly onto a quartz plate. The high solution-processability of ACQ@AIE-PMOs suggests their potential for practical solid-state lighting applications.

\section{Tuneable multicolour emission of R6G-doped AIE-PMOs}

To estimate the generality and versatility of our design concept for achieving the collaboration of AIE and ACQ dyes and constructing multicolour emission luminescent materials, R6G dye was also selected as an encapsulate. The PL spectra of AIE-PMO2 allow for partial spectral overlap with the absorption of R6G (Fig. S13 $\dagger$ ). This overlap suggests the possibility of FRET (a)

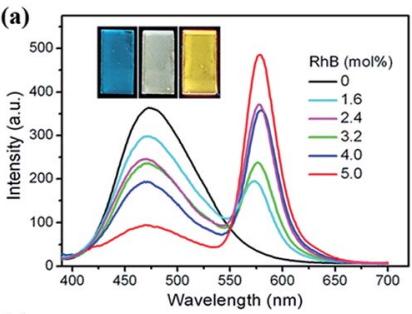

(c)

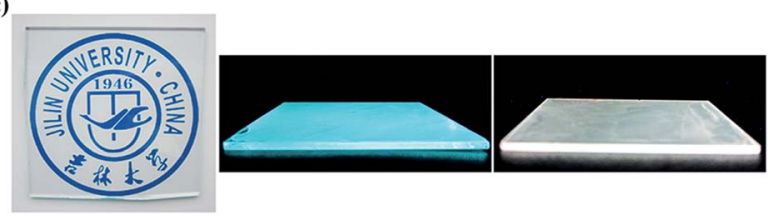

(b)

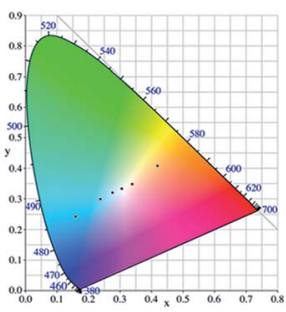

Fig. 5 (a) Fluorescence spectra of RhB@AIE-PMO films with different RhB content. (b) Corresponding emission colours of the films (marked by the dots) in the CIE 1931 chromaticity diagram. (c) PMO composite films coated on $10 \times 10 \mathrm{~cm}$ glass under sunlight (left), blue film on $10 \times$ $10 \mathrm{~cm}$ glass (middle) and white-light film on $5 \times 5 \mathrm{~cm}$ quartz (right) under $365 \mathrm{~nm}$ UV irradiation.

with R6G molecules as the acceptor and AIE-PMO2 as the donor. The energy transfer process from the AIE-PMO2 framework to the R6G dye inside the pore in the solid state was characterised by fluorescence spectroscopy (Fig. S14a $\dagger$ ). With increasing R6G content, the donor blue-light emission of AIE-PMO2 at $480 \mathrm{~nm}$ was quenched dramatically, whereas the acceptor emission at $560 \mathrm{~nm}$ of R6G increased gradually. The perceived emission colour of R6G-doped AIE-PMO2 varied from blue to yellow, as plotted in the CIE 1931 chromaticity diagram shown in Fig. S14b $\uparrow$. The fluorescence quantum yield increased from $20.0 \%$ to $38.0 \%$ and $46.9 \%$, when the R6G content was $0 \mathrm{~mol} \%, 0.23 \mathrm{~mol} \%$ and $0.68 \mathrm{~mol} \%$, respectively. These results strongly suggest that the excitation energy of the TPE units in the silica framework can also transfer to the R6G moieties in the solid state.

\section{Conclusions}

In summary, we have successfully demonstrated a facile strategy to achieve the effective coupling of AIE and ACQ dyes with exactly opposite photoluminescence behaviours by taking advantage of the unique structure of PMOs. The emission of the resulting ACQ@AIE-PMO composites can be fine-tuned over the entire visible spectrum by adjusting the content of encapsulated ACQ dyes. Significantly, highly pure solid-state white light can be achieved with a quantum yield as high as $49.6 \%$. Furthermore, white-emitting ACQ@AIE-PMO transparent films can be fabricated on a large scale through sol-gel polycondensation. The considerable flexibility of the rational combination of a great number of AIE chromophores and a wide range of ACQ dyes may facilitate the design of a variety of high-efficiency and tuneable multicolour emission PMOs. This work may create a new perspective for the exploration of novel high-performance solid luminescent materials that are easy-to-fabricate for various fluorescence-based applications in solid-state lighting, biomedicine and others. 


\section{Acknowledgements}

This work is supported by the State Basic Research Project of China (Grant No. 2011CB808703) and the National Natural Science Foundation of China (Grant No. 91122029 and 21320102001).

\section{Notes and references}

1 (a) R. Weissleder, C. H. Tung, U. Mahmood and A. Bogdanov, Nat. Biotechnol., 1999, 17, 375; (b) Y. Urano, D. Asanuma, Y. Hama, Y. Koyama, T. Barrett, M. Kamiya, T. Nagano, T. Watanabe, A. Hasegawa, P. L. Choyke and H. Kobayashi, Nat. Med., 2009, 15, 104; (c) H. N. Kim, Z. Q. Guo, W. H. Zhu, J. Yoon and H. Tian, Chem. Soc. Rev., 2011, 40, 79; (d) A. Razgulin, N. Ma and J. H. Rao, Chem. Soc. Rev., 2011, 40, 4186; (e) J. Chan, S. C. Dodani and C. J. Chang, Nat. Chem., 2012, 4, 973.

2 S. W. Thomas III, G. D. Joly and T. M. Swager, Chem. Rev., 2007, 107, 1339.

3 (a) J. D. Luo, Z. L. Xie, J. W. Y. Lam, L. Cheng, H. Y. Chen, C. F. Qiu, H. S. Kwok, X. W. Zhan, Y. Q. Liu, D. B. Zhu and B. Z. Tang, Chem. Commun., 2001, 1740; (b) Y. N. Hong, J. W. Y. Lam and B. Z. Tang, Chem. Soc. Rev., 2011, 40, 5361; (c) D. Ding, K. Li, B. Liu and B. Z. Tang, Acc. Chem. Res., 2013, 46, 2441; (d) Y. P. Zhang, D. D. Li, Y. Li and J. H. Yu, Chem. Sci., 2014, 5, 2710; (e) C. X. Shi, Z. Q. Guo, Y. L. Yan, S. Q. Zhu, Y. S. Xie, Y. S. Zhao, W. H. Zhu and H. Tian, ACS Appl. Mater. Interfaces, 2013, 5, 192; (f) A. D. Shao, Y. S. Xie, S. J. Zhu, Z. Q. Guo, S. Q. Zhu, J. Guo, P. Shi, T. D. James, H. Tian and W. H. Zhu, Angew. Chem., Int. Ed., 2015, 54, 7275.

4 (a) J. Huang, N. Sun, Y. Q. Dong, R. L. Tang, P. Lu, P. Cai, Q. Q. Li, D. G. Ma, J. G. Qin and Z. Li, Adv. Funct. Mater., 2013, 23, 2329; (b) Y. N. Hong, J. W. Y. Lam and B. Z. Tang, Chem. Commun., 2009, 4332.

5 (a) W. Z. Yuan, P. Lu, S. M. Chen, J. W. Y. Lam, Z. M. Wang, Y. Liu, H. S. Kwok, Y. G. Ma and B. Z. Tang, Adv. Mater., 2010, 22, 2159; (b) Q. L. Zhao, X. A. Zhang, Q. Wei, J. Wang, X. Y. Shen, A. J. Qin, J. Z. Sun and B. Z. Tang, Chem. Commun., 2012, 48, 11671; (c) A. Ozawa, A. Shimizu, R. Nishiyabu and Y. Kubo, Chem. Commun., 2015, 51, 118; (d) G. Chen, W. B. Li, T. R. Zhou, Q. Peng, D. Zhai, H. X. Li, W. Z. Yuan, Y. M. Zhang and B. Z. Tang, Adv. Mater., DOI: 10.1002/adma.201501981.
6 (a) M. Roushan, X. Zhang and J. Li, Angew. Chem., Int. Ed., 2012, 51, 436; (b) T. Wang, V. Chirmanov, W. H. M. Chiu and P. V. Radovanovic, J. Am. Chem. Soc., 2013, 135, 14520; (c) C. Y. Sun, X. L. Wang, X. Zhang, C. Qin, P. Li, Z. M. Su, D. X. Zhu, G. G. Shan, K. Z. Shao, H. Wu and J. Li, Nat. Commun., 2013, 4, 2717; (d) M. M. Shang, C. X. Li and J. Lin, Chem. Soc. Rev., 2014, 43, 1372; (e) E. R. Dohner, E. T. Hoke and H. I. Karunadasa, J. Am. Chem. Soc., 2014, 136, 1718.

7 (a) P. N. Minoofar, B. S. Dunn and J. I. Zink, J. Am. Chem. Soc., 2005, 127, 2656; (b) F. Hoffmann and M. Fröba, Chem. Soc. Rev., 2011, 40, 608; (c) A. Thomas, Angew. Chem., Int. Ed., 2010, 49, 8328; (d) Y. Yamamoto, H. Takeda, T. Yui, Y. Ueda, K. Koike, S. Inagaki and O. Ishitani, Chem. Sci., 2014, 5, 639; (e) T. Tani, N. Mizoshita and S. Inagaki, J. Mater. Chem., 2009, 19, 4451.

8 (a) N. Mizoshita, T. Tani and S. Inagaki, Chem. Soc. Rev., 2011, 40, 789; (b) S. H. Cheng, C. C. Hsiehc, N. T. Chen, C. H. Chu, C. M. Huang, P. T. Chou, F. G. Tseng, C. S. Yang, C. Y. Mou and L. W. Lo, Nano Today, 2011, 6, 552; (c) Y. Li, F. Auras, F. Löbermann, M. Döblinger, J. Schuster, L. Peter, D. Trauner and T. Bein, J. Am. Chem. Soc., 2013, 135, 18513.

9 Y. Goto, N. Mizoshita, O. Ohtani, T. Okada, T. Shimada, T. Tani and S. Inagaki, Chem. Mater., 2008, 20, 4495.

10 (a) D. D. Li, J. H. Yu and R. R. Xu, Chem. Commun., 2011, 47, 11077; (b) D. D. Li, J. Z. Liu, R. T. K. Kwok, Z. Q. Liang, B. Z. Tang and J. H. Yu, Chem. Commun., 2012, 48, 7167.

11 (a) M. Waki, Y. Maegawa, K. Hara, Y. Goto, S. Shirai, Y. Yamada, N. Mizoshita, T. Tani, W. J. Chun, S. Muratsugu, M. Tada, A. Fukuoka and S. Inagaki, J. Am. Chem. Soc., 2014, 136, 4003; (b) K. E. Sapsford, L. Berti and I. L. Medintz, Angew. Chem., Int. Ed., 2006, 45, 4562; (c) B. J. Scott, M. H. Bartl, G. Wirnsberger and G. D. Stucky, J. Phys. Chem. A, 2003, 107, 5499; (d) N. Mizoshita, Y. Goto, Y. Maegawa, T. Tani and S. Inagaki, Chem. Mater., 2010, 22, 2548.

12 (a) G. W. Zhou, Y. J. Chen, J. H. Yang and S. H. Yang, J. Mater. Chem., 2007, 17, 2839; (b) Y. Guillemin, J. Ghanbaja, E. Aubert, M. Etienne and A. Walcarius, Chem. Mater., 2014, 26, 1848.

13 E. Fuente, J. A. Menéndez, M. A. Díez, D. Suárez and M. Montes-Morán, J. Phys. Chem. B, 2003, 107, 6350. 